\title{
Yuanji Cheng
}

On positive solutions of quasilinear elliptic systems

Czechoslovak Mathematical Journal, Vol. 47 (1997), No. 4, 681-687

Persistent URL: http://dml.cz/dmlcz/127386

\section{Terms of use:}

(C) Institute of Mathematics AS CR, 1997

Institute of Mathematics of the Czech Academy of Sciences provides access to digitized documents strictly for personal use. Each copy of any part of this document must contain these Terms of use.

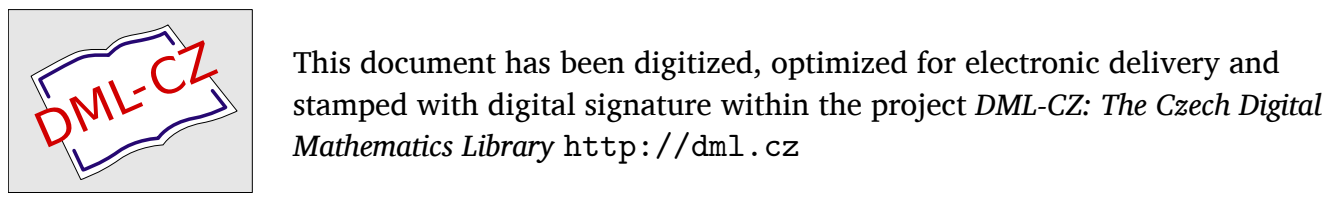




\title{
ON POSITIVE SOLUTIONS OF QUASILINEAR ELLIPTIC SYSTEMS
}

\author{
YUANJI CHENG, Luleå
}

(Received March 3, 1995)

Abstract. In this paper, we consider the existence and nonexistence of positive solutions of degenerate elliptic systems

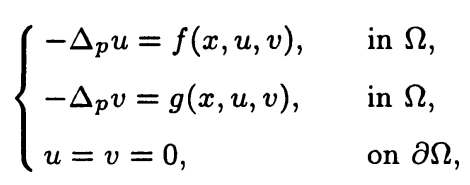

where $-\Delta_{p}$ is the $p$-Laplace operator, $p>1$ and $\Omega$ is a $C^{1, \alpha}$-domain in $\mathbb{R}^{n}$. We prove an analogue of $[7,16]$ for the eigenvalue problem with $f(x, u, v)=\lambda_{1} v^{p-1}, g(x, u, v)=\lambda_{2} u^{p-1}$ and obtain a non-existence result of positive solutions for the general systems.

Keywords: Eigenvalue problem, Degenerate elliptic operator, Nonlinear systems, Positive solutions.

1. Let $\Omega$ be a bounded $C^{1, \alpha}$-domain in $\mathbb{R}^{n}$, and $-\Delta_{p}$ the $p$-Laplace operator, $p>1$. In this paper, we are concerned with positive solutions of the elliptic system

$$
\left\{\begin{aligned}
-\Delta_{p} u=f(x, u, v), & & \text { in } \Omega, \\
-\Delta_{p} v=g(x, u, v), & & \text { in } \Omega, \\
u=v=0, & & \text { on } \partial \Omega .
\end{aligned}\right.
$$

For semilinear equations, the above problem is mentioned in [12] as an open problem and has been studied, for example, in [4] on the convex domain, in [14] for a ball, and in [7] on an annulus for systems with more than two equations. The quasilinear elliptic systems on the unit ball were investigated in [5] by an ODE approach. To understand the quasilinear system (1), we found that there is a big difference between semilinear and quasilinear systems, which is created by the nonlinearity of the $p$ Laplace operator. For instance, it is much harder to deduce the $L^{\infty}$-boundedness 
for positive solutions of (1), partly due to the fact there is no corresponding Rellich identity $[13,16]$, than in the semilinear systems, where linearity of the Laplacian plays an important role. Further, some simple facts about non-existence of positive solutions of semilinear systems become very delicate to handle in the quasilinear version (1). Anyway, our proofs are new even for the semilinear equations.

In the sequel, we denote by $\mu(p)>0, \varphi(x)>0$ the first eigenvalue and the corresponding normalized eigenfunction of the $p$-Laplace operator $-\Delta_{p}$ [11]. For a given uniformly elliptic operator $L=-\sum_{i, j} \partial_{i}\left(a_{i j}(x) \partial_{j}\right)$ we denote the eigenvalues and the corresponding eigenfunctions [6] by $\mu_{k}, \psi_{k}(x), k=1,2, \ldots$.

First we consider the following linear eigenvalue problem for $u, v \in W_{0}^{1,2}(\Omega)$ :

$$
\left\{\begin{array}{l}
L u=\alpha u+\lambda_{1} v \\
L v=\lambda_{2} u+\beta v
\end{array}\right.
$$

where $\alpha, \lambda_{1}, \lambda_{2}, \beta \in \mathbb{R}$.

Theorem 1. 1) If $\alpha, \beta<\mu_{1}$ then (2) has positive solutions if and only if $\lambda_{1}>0$ and $\lambda_{1} \lambda_{2}=\left(\mu_{1}-\alpha\right)\left(\mu_{1}-\beta\right)$.

2) The system (2) has nontrivial solutions if and only if $\lambda_{1} \lambda_{2}=\left(\mu_{k}-\alpha\right)\left(\mu_{k}-\beta\right)$ for some $k \geqslant 1$ and the solutions $u, v$ belong to the eigenspace of $\mu_{k}$.

As a corollary of Theorem 1, we consider the following elliptic systems [10] on $W_{0}^{1,2}(\Omega)$ :

$$
\left\{\begin{array}{l}
-\alpha \Delta u-\beta \Delta v=\lambda_{1} v, \\
-\beta \Delta u-\delta \Delta v=\lambda_{2} u,
\end{array}\right.
$$

where $\alpha, \beta, \lambda, \delta, \mu$ are constants and $\alpha>0, \alpha \delta-\beta^{2}>0$.

Corollary 1. The system (3) has a pair of positive solutions if and only if $\lambda_{1}>$ $\beta \mu_{1}, \alpha \delta \mu_{1}^{2}=\left(\lambda_{1}-\beta \mu_{1}\right)\left(\lambda_{2}-\beta \mu_{1}\right)$ and the solutions are of the form $u=a \psi_{1}(x)$, $v=b \psi_{1}(x)$.

For the special quasilinear elliptic system: $u, v \in W_{0}^{1, p}(\Omega)$,

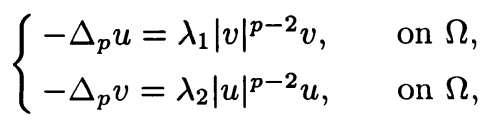

we have

Theorem 2. 1) The system (4) has positive solutions if and only if $\lambda_{1}>0$, $\lambda_{1} \lambda_{2}=\mu(p)^{2}$ and the solutions are given by $u=c_{1} \varphi(x), v=c_{2} \varphi(x), c_{1}>0$, $c_{2}=c_{1}\left(\lambda_{2} / \lambda_{1}\right)^{1 / 2(p-1)}$. 
2) If $\lambda_{1} \lambda_{2} \geqslant 0$, then (4) has nontrivial solutions if and only if $\sqrt{\lambda_{1} \lambda_{2}}$ is an eigenvalue of $-\Delta_{p}$ and $u, v$ are the associated eigenfunctions.

Concerning the general system (1), we only have a non-existence result

Theorem 3. If there exist non-negative constants $\alpha, \beta, \lambda_{i}, i=1,2$ such that one of the following conditions is satisfied, then (1) has only trivial non-negative solution $u, v \leqslant k$ :

1) $\Lambda<\mu(p)$ and for all $(x, u, v) \in \Omega \times[0, k]^{2}$,

$$
f(x, u, v) \leqslant \alpha u^{p-1}+\lambda_{1} v^{p-1}, \quad g(x, u, v) \leqslant \lambda_{2} u^{p-1}+\beta v^{p-1} ;
$$

2) $\Lambda>\mu(p)$ and for all $(x, u, v) \in \Omega \times[0, k]^{2}$,

$$
f(x, u, v) \geqslant \alpha u^{p-1}+\lambda_{1} v^{p-1}, \quad g(x, u, v) \geqslant \lambda_{2} u^{p-1}+\beta v^{p-1},
$$

where $2 \Lambda=\alpha+\beta+\sqrt{(\alpha-\beta)^{2}+4 \lambda_{1} \lambda_{2}}$.

2. In this part, we prove a lemma, which is an improvement of the result in $[7,16]$. From now on we work on the Sobolev space $W_{0}^{1, p}(\Omega)$ with the norm $\|u\|^{p}=$ $\int_{\Omega}|\nabla u|^{p} \mathrm{~d} x$.

Lemma 1. If $\alpha_{0}>0$ is constant and $u, v$ are nonzero elements of $W_{0}^{1, p}(\Omega)$ satisfying

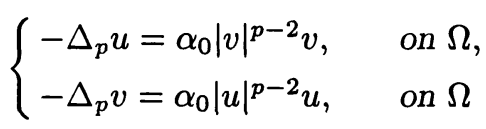

in the weak sense, then $u=v$ and $\alpha_{0}$ is an eigenvalue of $-\Delta_{p}$. Moreover, if $u \geqslant 0$ on $\Omega$, then $\alpha_{0}=\mu(p)$ and $u=v=c \varphi(x)$ for constant $c$.

$\operatorname{Pr}$ oof. If we choose $\psi=(u-v)_{+} \in W_{0}^{1, p}(\Omega)$ as a test function for (5), we see [8] that

$$
\begin{aligned}
& \int_{\{u>v\}}|\nabla u|^{p-2} \nabla u \cdot \nabla(u-v) \mathrm{d} x=\alpha_{0} \int_{\{u>v\}}|v|^{p-2} v(u-v) \mathrm{d} x, \\
& \int_{\{u>v\}}|\nabla v|^{p-2} \nabla v \cdot \nabla(u-v) \mathrm{d} x=\alpha_{0} \int_{\{u>v\}}|u|^{p-2} u(u-v) \mathrm{d} x .
\end{aligned}
$$

It follows from (6) and (7) that

$$
\begin{aligned}
& \int_{\{u>v\}}\left(|\nabla u|^{p-2} \nabla u-|\nabla v|^{p-2} \nabla v\right) \cdot \nabla(u-v) \mathrm{d} x \\
& =-\alpha_{0} \int_{\{u>v\}}\left(|u|^{p-2} u-|v|^{p-2} v\right)(u-v) \mathrm{d} x \leqslant 0,
\end{aligned}
$$


which implies that $u \leqslant v$ a.e. on $\Omega$. Similarly, it can be also shown that $u \geqslant v$ a.e. on $\Omega$. Consequently, $u=v$ and $\alpha_{0}$ is an eigenvalue of $-\Delta_{p}$. Since the first eigenvalue of $-\Delta_{p}$ is simple [11], it follows that $\alpha_{0}=\mu(p)$ in the case $u \geqslant 0$ or $v \geqslant 0$ on $\Omega$.

3. We shall prove Theorems 1 to 3 in this section.

Proof of Theorem 1 .

1) The sufficiency is obvious, since $\left(\psi_{1}(x),(\mu-\alpha) / \lambda_{1} \cdot \psi_{1}(x)\right)$ is a pair of positive solutions. It remains to show the necessity. First observe that if $(u, v)$ is a pair of positive solutions of (2), then $\lambda_{i}>0$, since $\alpha, \beta<\mu_{1}$ and $L-\alpha, L-\beta$ are positive operators. Further, choosing $c>0$ such that $\alpha+c \lambda_{1}=\lambda_{2} / c+\beta=\bar{\Lambda}$ and changing variables by $u_{0}=u, v_{0}=v / c$, we see that $u_{0}, v_{0}$ satisfy the system

$$
\left\{\begin{array}{l}
L u_{0}=\alpha u_{0}+c \lambda_{1} v_{0}, \\
L v_{0}=\lambda_{2} u_{0} / c+\beta v_{0} .
\end{array}\right.
$$

Now, define $w=\max \left\{u_{0}, v_{0}\right\}, w_{0}=\min \left\{u_{0}, v_{0}\right\}$, then we have

$$
\begin{gathered}
w+w_{0}=u_{0}+v_{0}, \quad w-w_{0}=\left|u_{0}-v_{0}\right|, \\
\left\{\begin{array}{l}
L u_{0}=\alpha u_{0}+c \lambda_{1} v_{0} \leqslant \bar{\Lambda} w, \\
L v_{0}=\lambda_{2} u_{0} / c+\beta v_{0} \leqslant \bar{\Lambda} w,
\end{array}\right.
\end{gathered}
$$

which implies [8] that $w$ satisfies

$$
L w \leqslant \bar{\Lambda} w
$$

Similarly, $w_{0}$ satisfies the inequality $L w_{0} \leqslant \bar{\Lambda} w_{0}$. Consequently,

$$
L\left(w-w_{0}\right) \leqslant \bar{\Lambda}\left(w-w_{0}\right) .
$$

If we use $\psi=w$ as a test function for the inequality (9), we get

$$
\int_{\Omega} a_{i j} w_{x_{i}} w_{x_{j}} \mathrm{~d} x \leqslant \bar{\Lambda} \int_{\Omega} w^{2} \mathrm{~d} x
$$

which implies that $\bar{\Lambda}=\mu_{1}, w=\gamma \psi_{1}(x)$ for some $\gamma>0$ because

$$
\mu_{1}=\inf \left\{\int_{\Omega} a_{i j} w_{x_{i}} w_{x_{j}} \mathrm{~d} x / \int_{\Omega} w^{2} \mathrm{~d} x, \quad 0 \neq w \in W_{0}^{0,1}(\Omega)\right\}
$$

and $\psi_{1}$ is the normalized minimizer [6]. In a similar way, we derive $w-w_{0}=\gamma_{0} \psi_{1}$ for some $\gamma_{0} \geqslant 0$. 
We are done if $\gamma_{0}=0$. If $\gamma_{0}>0$, then $u_{0}-v_{0}$ does not change its sign on $\Omega$ due to the fact that $\psi_{1}$ is positive on $\Omega$. Therefore, either $u_{0}-v_{0}=\gamma \psi_{1}$ or $v_{0}-u_{0}=\gamma_{0} \psi_{1}$. We may assume that $u_{0}-v_{0}=\gamma_{0} \psi_{1}$, then $u_{0}=\gamma \psi_{1}$ and $v_{0}=\left(\gamma-\gamma_{0}\right) \psi_{1}$. Via the first equation in (8), we deduce

$$
\mu_{1} \gamma=\alpha \gamma+\lambda_{1} c\left(\gamma-\gamma_{0}\right)=\bar{\Lambda} \gamma-\lambda_{1} c \gamma_{0}
$$

But $\bar{\Lambda}=\mu_{1}$, so we see from the above equation that $\lambda_{1} c \gamma_{0}=0$. This is a contradiction and the proof of 1) is complete.

2) Let $(\mathrm{u}, \mathrm{v})$ be a nontrivial solution of (2), then $u, v$ have the expansions [6] $u(x)=\sum_{k} a_{k} \psi_{k}(x), v=\sum_{k} b_{k} \psi_{k}(x)$, with $0 \neq\left\{a_{k}\right\}_{1}^{\infty},\left\{b_{k}\right\}_{1}^{\infty} \in \ell^{2}$. We obtain from (2) that $a_{k}, b_{k}$ solve the system of linear equations

$$
\left\{\begin{array}{l}
\mu_{k} a_{k}=\alpha a_{k}+\lambda_{1} b_{k}, \\
\mu_{k} b_{k}=\lambda_{2} a_{k}+\beta b_{k},
\end{array}\right.
$$

which has a nontrivial solution $\left(a_{k}, b_{k}\right)$ if and only if $\lambda_{1} \lambda_{2}=\left(\mu_{k}-\alpha\right)\left(\mu_{k}-\beta\right)$.

Proof of Theorem 2 .

1) First observe by the positivity of the $p$-Laplace operator [9] that if (3) has a pair of positive solutions then $\lambda_{i}>0, i=1,2$. The assertion follows from Lemma and a rescaling argument.

2) Assuming that $\sqrt{\lambda_{1} \lambda_{2}}$ is an eigenvalue of $-\Delta_{p}$, then $\left(\left|\lambda_{1}\right|^{\frac{1}{2(p-1)}} \psi(x)\right.$, $\left|\lambda_{2}\right|^{\frac{1}{2(p-1)}} \psi(x)$ ) is a pair of solutions of (3), where $\psi$ is an associated eigenfunction of $\sqrt{\lambda_{1} \lambda_{2}}$. On the other hand, if $\lambda_{1} \lambda_{2} \geqslant 0$ and $(u, v)$ is a nontrivial solution of (3), then we derive by the Sobolev embedding theorem that $\lambda_{1} \lambda_{2}>0$ and both $u$ and $v$ are nonzero elements in $W_{0}^{1, p}(\Omega)$. Furthermore, changing the variables by

$$
u_{0}=\left|\lambda_{1}\right|^{\frac{1}{2(p-1)}} u, \quad v_{0}=\left|\lambda_{2}\right|^{\frac{1}{2(p-1)}} v,
$$

we see that $\left(u_{0}, v_{0}\right)$ satisfies the system (5) with $\alpha_{0}=\sqrt{\lambda_{1} \lambda_{2}}$ and hence the conclusion is true via Lemma.

Open problem: Is there a positive number $\alpha>0$ such that the system

$$
\left\{\begin{aligned}
-\Delta_{p} u & =\alpha|v|^{p-2} v, & & \text { on } \Omega, \\
-\Delta_{p} v & =-\alpha|u|^{p-2} u, & & \text { on } \Omega
\end{aligned}\right.
$$

has a pair of nontrivial solutions $u, v \in W_{0}^{1, p}(\Omega)$ ? 
Pro of of Theorem 3. Since the case when $\lambda_{1} \lambda_{2}=0$ is easy, we may assume $\lambda_{1} \lambda_{2}>0$. Supposing that (1) has a pair of positive solutions $u(x), v(x) \leqslant k$ a.e. on $\Omega$, we change variables by

$$
u=u_{0}, \quad v=c v_{0}, \quad \text { where } \quad 2 \lambda_{1} c^{p-1}=\beta-\alpha+\sqrt{(\beta-\alpha)^{2}+4 \lambda_{1} \lambda_{2}} .
$$

Let $\widehat{w}=\max \left\{u_{0}, v_{0}\right\}, \widetilde{w}=\min \left\{u_{0}, v_{0}\right\}$, then $\widehat{w}, \widetilde{w} \leqslant k$ a.e. on $\Omega$. If the condition 1$)$ in Theorem 3 holds, we have $\Lambda<\mu(p)$ and

$$
\begin{aligned}
& -\Delta_{p} u_{0}=f(x, u, v) \leqslant \alpha u_{0}^{p-1}+\lambda_{1} c^{p-1} v_{0}^{p-1} \leqslant \Lambda \widehat{w}^{p-1}, \\
& -\Delta_{p} v_{0}=c^{1-p} g(x, u, v) \leqslant c^{1-p} \lambda_{2} u_{0}^{p-1}+\beta v_{0}^{p-1} \leqslant \Lambda \widehat{w}^{p-1} .
\end{aligned}
$$

From these two inequalities we get [8] that $\widehat{w}$ satisfies the inequality $-\Delta_{p} w \leqslant \Lambda w^{p-1}$ in the weak sense, which implies that $\|\widehat{w}\|^{p} \leqslant \Lambda\|\widehat{w}\|_{L^{p}}^{p}$. But this inequality has only the trivial solution $w=0$, if $\Lambda<\mu(p)$.

It the condition 2) satisfied, then $\Lambda>\mu(p)$. Analogously, we can show that $\widetilde{w}$ satisfies $-\Delta_{p} w \geqslant \Lambda w^{p-1}$ in the weak sense.

Define $\tau=(\Lambda / \mu(p))^{1 /(p-1)}>1$ and choose $t>0$ such that $t \varphi(x) \leqslant \tau \widetilde{w}(x)$ on $\Omega$, which is possible due to the fact that $\widetilde{w}, \varphi>0$ on $\Omega$, and $\frac{\partial \widetilde{w}}{\partial \nu}, \frac{\partial \varphi}{\partial \nu}>0$ on $\partial \Omega$ [15]. Denoting $\varphi_{1}(x)=t \varphi(x)$, and then choosing $\left(\varphi_{1}-\widetilde{w}\right)_{+}$as a test function both for the differential inequality $-\Delta_{p} w \geqslant \Lambda w^{p-1}$ and the equation $-\Delta_{p} \varphi_{1}=\mu(p) \varphi_{1}^{p-1}$, we obtain

$$
\begin{aligned}
& \int_{\left\{\varphi_{1}>\widetilde{w}\right\}}|\nabla \widetilde{w}|^{p-2} \nabla \widetilde{w} \cdot \nabla\left(\varphi_{1}-\widetilde{w}\right) \mathrm{d} x \geqslant \Lambda \int_{\left\{\varphi_{1}>\widetilde{w}\right\}} \widetilde{w}^{p-1}\left(\varphi_{1}-\widetilde{w}\right) \mathrm{d} x, \\
& \int_{\left\{\varphi_{1}>\tilde{w}\right\}}\left|\nabla \varphi_{1}\right|^{p-2} \nabla \varphi_{1} \cdot \nabla\left(\varphi_{1}-\widetilde{w}\right) \mathrm{d} x=\mu(p) \int_{\left\{\varphi_{1}>\widetilde{w}\right\}} \varphi_{1}^{p-1}\left(\varphi_{1}-\widetilde{w}\right) \mathrm{d} x .
\end{aligned}
$$

Consequently,

$$
\begin{aligned}
0 & \geqslant \int_{\left\{\varphi_{1}>\widetilde{w}\right\}}\left(|\nabla \widetilde{w}|^{p-2} \nabla \widetilde{w}-\left|\nabla \varphi_{1}\right|^{p-2} \nabla \varphi_{1}\right) \cdot \nabla\left(\varphi_{1}-\widetilde{w}\right) \mathrm{d} x \\
& \geqslant \int_{\left\{\varphi_{1}>\widetilde{w}\right\}}\left\{\Lambda \widetilde{w}^{p-1}-\mu(p) \varphi_{1}^{p-1}\right\}\left(\varphi_{1}-\widetilde{w}\right) \mathrm{d} x \\
& \geqslant \nu(p)^{-1} \int_{\left\{\varphi_{1}>\widetilde{w}\right\}}\left\{(\tau \widetilde{w})^{p-1}-\varphi_{1}^{p-1}\right\}\left(\varphi_{1}-\widetilde{w}\right) \mathrm{d} x \geqslant 0,
\end{aligned}
$$

because on the domain $\left\{x ; \varphi_{1}(x)>\widetilde{w}(x)\right\}$ the inequality $\left\{(\tau \widetilde{w})^{p-1}-\varphi_{1}^{p-1}\right\}\left(\varphi_{1}-\widetilde{w}\right)>$ 0 holds. Thus, we have $\varphi_{1} \leqslant \widetilde{w}$ a.e. on $\Omega$.

Applying the preceding trick once more to the eigenfunction $\tau \varphi_{1}(\leqslant \tau \widetilde{w})$ we obtain $\tau \varphi_{1} \leqslant \widetilde{w}$ on $\Omega$, too. An iteration process yields $\tau^{n} \varphi \leqslant \widetilde{w}$ on $\Omega$ for any integer $n \geqslant 1$. Letting $n$ tend to infinity, we deduce $\varphi \equiv 0$ on $\Omega$. This is absurd and the proof is done. 
Acknowledgement. The author would like to thank the referee for pointing out an error in the first version of the manuscript.

\section{References}

[1] $Y$. Cheng: On the existence of radial solutions of a nonlinear elliptic BVP in an annulus. Math. Nachr. 165 (1994), 61-77.

[2] $Y$. Cheng: On the existence of radial solutions of a nonlinear elliptic equation on the unit ball. Nonlinear Analysis, TMA, 24:3 (1995), 287-307.

[3] Y. Cheng: An eigenvalue problem for a quasilinear elliptic equation. U.U.M.D. Report NO:19, 1994.

[4] Ph. Clément, D. de Figueiredo, E. Mitidieri: Positive solutions of semilinear elliptic systems. Comm. P.D.E. 17 (1992), 923-940.

[5] Ph. Clément, R. Manásevich, E. Mitidieri: Positive solutions of quasilinear elliptic systems via blow up. Comm. P.D.E. 18 (1993), 2071-2116.

[6] R. Courant, D. Hilbert: Methods of Mathematical Physics II. Interscience, 1953.

[7] R. Dalmasso: Positive solutions of nonlinear elliptic systems. Annl. Pol. Math. LVIII.2 (1993), 201-213.

[8] D. Gilbarg, N. S. Trudinger: Elliptic Partial Differential Equations of Second Order. Second edition, Springer-Verlag, 1992.

[9] J. Heinonen, T. Kilpelaninen, O. Martio: Nonlinear Potential Theory of Degenerate Elliptic Equations. Oxford, University Press, 1993.

[10] $H$. A. Levine: The role of critical exponents in blow-up theorems. SIAM Review 32:2 (1990), 262-288.

[11] P. Lindqvist: On the equation $\operatorname{div}\left(|\nabla u|^{p-2} \nabla u\right)+\lambda|u|^{p-2} u=0$. Proc. Amer. Math. Soci. 109 (1990), 157-164.

[12] P. Lions: On the existence of positive solutions of semilinear elliptic equations. SIAM Review 24 (1982), 441-467.

[13] M. Mitidieri: A Rellich type identity and applications. Comm. P.D.E. 18 (1993), 125-151.

[14] L. Peletier, R.C.M. van der Vorst: Existence and nonexistence of positive solutions of nonlinear elliptic systems and the biharmonic equations. Diff Interg. Equa. 5 (1992), 747-767.

[15] S. Sakaguchi: Concavity property of solutions to some degenerate quasilinear elliptic Dirichlet problems. Ann. Scuola. Norm. Pisa 14 (1987), 403-421.

[16] R.C.M. van der Vorst: Variational identities and applications to differential systems. Arch. rational Mech. Anal. 116c (1991), 375-398.

Author's address: Department of Mathematics, Luleå University of Technology, 97187 Luleå, Sweden, email: cheng@sm.luth.se. 